\title{
THE SOLUTION OF CERTAIN SIMULTANEOUS PAIRS OF DUAL INTEGRAL EQUATIONS $\dagger$
}

\author{
by M. LOWENGRUB
}

(Received 27 April, 1967)

1. Introduction. In the analysis of mixed boundary value problems by Hankel transforms, one often encounters dual integral equations of the form

$$
\begin{aligned}
& \int_{0}^{\infty} w_{1}(x) J_{\mu}(x y) \psi(x) d x=f(y) \quad\left(y \in I_{1}\right), \\
& \int_{0}^{\infty} w_{2}(x) J_{v}(x y) \psi(x) d x=g(y) \quad\left(y \in I_{2}\right),
\end{aligned}
$$

where $I_{1}=(0,1), I_{2}=(1, \infty) ; w_{1}(x), w_{2}(x)$ are weight functions, $\psi(x)$ is the unknown function, and $f(y), g(y)$ are functions continuously differentiable on $I_{1}$ and $I_{2}$ respectively. Many successful attempts have been made to solve (1.1) and (1.2). These are all discussed in a recent book by Sneddon [7]. As pointed out in a recent paper by Erdogan and Bahar [4], in mixed boundary value problems of semi-infinite domains involving more than one unknown function such as those arising in elastostatics, viscoelasticity, and electrostatics, the formulation will lead to a system of simultaneous dual integral equations which is a generalization of (1.1) and (1.2). These equations may be expressed as follows:

$$
\begin{aligned}
& \mathscr{H}_{\mu_{i}}\left\{\sum_{j=1}^{n} w_{i j}(t) \psi_{j}(t) ; x\right\}=f_{i}(x) \quad\left(x \in I_{1}\right), \\
& \mathscr{H}_{v_{i}}\left\{\sum_{j=1}^{n} v_{i j}(t) \psi_{j}(t) ; x\right\}=g_{i}(x) \quad\left(x \in I_{2}\right),
\end{aligned}
$$

with $i=1,2, \ldots, n$, where we use the notation

$$
\mathscr{H}_{\eta}\{\psi(t) ; x\}=\int_{0}^{\infty} t \psi(t) J_{\eta}(x t) d t
$$

Erdogan and Bahar [4] solve (1.3) and (1.4) by reducing them to an infinite set of algebraic equations, using a natural generalisation of the method developed by Tranter [9] for dual integral equations. In this note, we pay particular attention to the cases which are of most physical interest-namely $w_{i j}(t)=t^{-2 \alpha} a_{i j}$ and $v_{i j}(t)=t^{-2 \beta} b_{i j}, a_{i j}$ and $b_{i j}$ being constants.

By using the Erdelyi-Kober operators of fractional integration described in Erdelyi and Sneddon [3], we are able to solve the equations in a fairly straightforward manner. In $\$ 3$ and 4 , we show all the details for the cases of two and three sets of equations while in $\S 5$ we outline the procedure for $n$ pairs of simultaneous dual integral equations.

† This research was supported in part by the United States Air Force under grant AF-AROSR 435-63, with Duke University, monitored by the Air Force Office of Scientific Research and in part by a National Science Foundation Science Faculty Fellowship. 
If we make the substitutions $w_{i j}(t)=t^{-2 \alpha} a_{i j}, v_{i j}(t)=t^{-2 \beta} b_{i j}$, we find that equations (1.3) and (1.4), assuming the validity of an interchange of order of summation and integration, may be written in the form:

$$
\begin{aligned}
& \sum_{j=1}^{n} a_{i j} \mathscr{H}_{\mu_{i}}\left[t^{-2 \alpha} \psi_{j}(t) ; x\right]=f_{i}(x) \quad\left(x \in I_{1}\right), \\
& \sum_{j=1}^{n} b_{i j} \mathscr{H}_{v_{i}}\left[t^{-2 \beta} \psi_{j}(t) ; x\right]=g_{i}(x) \quad\left(x \in I_{2}\right),
\end{aligned}
$$

with $i=1,2, \ldots, n$. Erdogan and Bahar [4] show that (1.5) and (1.6) may be reduced to the pair of equations

where

$$
\begin{aligned}
\sum_{j=1}^{n} c_{i j} \mathscr{H}_{\mu_{i}}\left[t^{-2 \alpha} \phi_{j}(t) ; x\right] & =p_{i}(x) & & \left(x \in I_{1}\right), \\
\mathscr{H}_{v_{i}}\left[t^{-2 \beta} \phi_{i}(t) ; x\right] & =0 & & \left(x \in I_{2}\right),
\end{aligned}
$$

$$
\left.\begin{array}{rl}
\phi_{i}(t) & =\sum_{j=1}^{n} b_{i j} \psi_{j}(t)-\tau_{i}(t), \\
\tau_{i}(t) & =t^{2 \beta} \int_{1}^{\infty} x g_{i}(x) J_{v_{i}}(x t) d x, \\
p_{i}(x) & =f_{i}(x)-\sum_{j=1}^{n} c_{i j} \mathscr{H}_{\mu_{i}}\left[t^{-2 \alpha} \tau_{j}(t) ; x\right],
\end{array}\right\}
$$

with $\left(c_{i j}\right)=C=A B^{-1}, A=\left(a_{i j}\right), B=\left(b_{i j}\right), i, j=1,2, \ldots, n$. Hence we shall only discuss the solution to (1.7) and (1.8), as the solution to the pair (1.5), (1.6) follows immediately from (1.9).

It should be pointed out that the pair of simultaneous dual integral equations considered by Westman [10], are a special case of (1.5), (1.6), namely that in which $n=2, \mu_{1}=v_{1}=v+2$, $\mu_{2}=v_{2}=v, a_{12}=a_{21}=a_{22}=b_{12}=b_{22}=1$. His method involves a straightforward generalization of the techniques used by Copson [2] and Lowengrub and Sneddon [5] to solve dual integral equations.

2. The Erdelyi-Kober and modified Hankel operators. Throughout this note, we shall make use of various operators of fractional integration whose properties are discussed in Erdelyi and Sneddon [5]. If $\alpha>0, \eta>-\frac{1}{2}$, we define the operator $I_{\eta, \alpha}$ as follows:

$$
I_{\eta, \alpha} f(x)=\frac{2 x^{-2 \alpha-2 \eta}}{\Gamma(\alpha)} \int_{0}^{x}\left(x^{2}-u^{2}\right)^{\alpha-1} u^{2 \eta+1} f(u) d u .
$$

$I_{\eta, 0}$ is the identity operator and, if $-1<\alpha<0$, we define $I_{\eta, \alpha}$ by the relation

$$
I_{\eta, \alpha} f(x)=\frac{x^{-2 \alpha-2 \eta-1}}{\Gamma(\alpha+1)} \frac{d}{d x}\left[\int_{0}^{x}\left(x^{2}-u^{2}\right)^{\alpha} u^{2 \eta+1} f(u) d u\right]
$$


Similarly, if $\alpha>0, \eta>-\frac{1}{2}$, we define the operator $K_{\eta, \alpha}$ by the equation

$$
K_{\eta, \alpha} f(x)=\frac{2 x^{2 \eta}}{\Gamma(\alpha)} \int_{x}^{\infty}\left(u^{2}-x^{2}\right)^{\alpha-1} u^{-2 \alpha-2 \eta+1} f(u) d u,
$$

while $K_{\eta, 0}$ is the identity operator and, for $-1<\alpha<0$, we define $K_{\eta, \alpha}$ by the relation

$$
K_{\eta, \alpha} f(x)=\frac{-x^{-2 \eta-1}}{\Gamma(1+\alpha)} \frac{d}{d x}\left[\int_{x}^{\infty}\left(u^{2}-x^{2}\right)^{\alpha} u^{-2 \alpha-2 \eta+1} f(u) d u\right] .
$$

The modified operator of Hankel transforms is defined by the formula

$$
S_{\eta, \alpha} f(x)=2^{\alpha} x^{-\alpha} \int_{0}^{\infty} t^{1-\alpha} J_{2 \eta+\alpha}(x t) f(t) d t .
$$

Erdelyi and Sneddon [3] show that the following relations connecting these operators hold:

$$
\begin{gathered}
I_{\eta+\alpha, \beta} S_{\eta, \alpha}=S_{\eta, \alpha+\beta}, \\
S_{\eta+\alpha, \beta} I_{\eta, \alpha}=S_{\eta, \alpha+\beta}, \\
K_{\eta, \alpha} S_{\eta+\alpha, \beta}=S_{\eta, \alpha+\beta}, \\
S_{\eta, \alpha} K_{\eta+\alpha, \beta}=S_{\eta, \alpha+\beta}, \\
S_{\eta+\alpha, \beta} S_{\eta, \alpha}=I_{\eta, \alpha+\beta}, \\
S_{\eta, \alpha} S_{\eta+\alpha, \beta}=K_{\eta, \alpha+\beta} .
\end{gathered}
$$

The inverse operators are given by

$$
\left.\begin{array}{c}
I_{\eta, \alpha}^{-1}=I_{\eta+\alpha,-\alpha}, \\
K_{\eta, \alpha}^{-1}=K_{\eta+\alpha,-\alpha}, \\
S_{\eta, \alpha}^{-1}=S_{\eta+\alpha,-\alpha} .
\end{array}\right\}
$$

In addition, we shall have occasion to use the following identity connecting $S, I$ and $K$ :

$$
S_{\sigma, \tau}=S_{\sigma+\gamma, \tau-\beta-\gamma} K_{\sigma+\tau-\beta, \beta} I_{\sigma, \gamma}
$$

3. Two pairs of dual equations. In this section, we shall consider the solution to the set of equations

$$
\begin{aligned}
S_{\frac{1}{2} \mu_{i}-\alpha, 2 \alpha}\left[\sum_{j=1}^{2} c_{i j} \phi_{j}(x)\right] & =p_{i}(x) & \left(x \in I_{1}\right), \\
S_{\frac{1}{2} v_{i}-\beta, 2 \beta} \phi_{i}(x) & =0 & \left(x \in I_{2}\right),
\end{aligned}
$$

$i=1,2, I_{1}=(0,1), I_{2}=(1, \infty)$. 
Following the techniques of Copson [2] and Peters [6] for dual integral equations, we set

$$
\begin{aligned}
& \phi_{1}(x)=S_{ \pm v_{1}+\beta, t\left(\mu_{1}-v_{1}\right)-\alpha-\beta} h_{1}(x)+S_{ \pm v_{2}+\beta, t\left(\mu_{2}-v_{2}\right)-\alpha-\beta} h_{2}(x), \\
& \phi_{2}(x)=S_{t v_{2}+\beta, t\left(\mu_{2}-v_{2}\right)-\alpha-\beta} h_{3}(x),
\end{aligned}
$$

where $h_{i}(x)=0, x \in I_{2}, i=1,2,3$.

From (2.13), it is clear that the representation (3.3) and (3.4) satisfies equation (3.2). Substituting into the relations given by (3.1), we find that the case $i=1$ yields

$$
\begin{array}{r}
I_{t v_{1}+\beta, \xi\left(\mu_{1}-v_{1}\right)+\alpha-\beta}\left[c_{11} h_{1}(x)\right]+S_{\frac{1}{2} \mu_{1}-\alpha, 2 \alpha} S_{\frac{1}{2} v_{2}+\beta, \xi\left(\mu_{2}-v_{2}\right)-\alpha-\beta}\left[c_{11} h_{2}(x)+c_{12} h_{3}(x)\right]=p_{1}(x) . \\
\left(x \in I_{1}\right)
\end{array}
$$

If we choose $h_{2}$ and $h_{3}$ such that

$$
c_{11} h_{2}(x)+c_{12} h_{3}(x) \equiv 0 \quad\left(x \in I_{1} \cup I_{2}\right),
$$

then it is clear that

$$
h_{1}(x)=c_{11}^{-1} I_{\frac{1}{2} \mu_{1}+\alpha,-\frac{1}{t}\left(\mu_{1}-v_{1}\right)-\alpha+\beta} p_{1}(x),
$$

provided that $c_{11} \neq 0$.

Substitution of (3.3) and (3.4) into (3.1) for $i=2$ yields the following relation:

$$
I_{\ddagger v_{2}+\beta, \xi\left(\mu_{2}-v_{2}\right)+\alpha-\beta}\left[c_{21} h_{2}(x)+c_{22} h_{3}(x)\right]=p_{2}(x)-c_{21} S_{\frac{1}{2} \mu_{2}-\alpha, 2 \alpha} S_{\frac{1}{\ddagger} v_{1}+\beta, \xi\left(\mu_{1}-v_{1}\right)-\alpha-\beta} h_{1}(x) \text {. }
$$

Inversion of the preceding equation with the use of (3.6) and (2.7) yields

$$
\begin{aligned}
c_{21} h_{2}(x)+c_{22} h_{3}(x)=I_{\ddagger \mu_{2}+\alpha,-\frac{1}{t}\left(\mu_{2}-v_{2}\right)-\alpha+\beta} & p_{2}(x) \\
& -c_{21} S_{\ddagger \mu_{2}-\alpha,-\frac{1}{t}\left(\mu_{2}-v_{2}\right)+\alpha+\beta} S_{\frac{1}{2} v_{1}+\beta,\left\{\left(\mu_{1}-v_{1}\right)-\alpha-\beta\right.} h_{1}(x) .
\end{aligned}
$$

Thus we find that

$$
\begin{aligned}
h_{3}(x)=\frac{c_{11}}{c_{22} c_{11}-c_{21} c_{12}} I_{j \mu_{2}+\alpha,-\frac{1}{j}\left(\mu_{2}-v_{2}\right)-\alpha+\beta} p_{2}(x) \\
\quad-\frac{c_{21} c_{11}}{c_{22} c_{11}-c_{21} c_{12}} S_{j \mu_{2}-\alpha_{1}-f\left(\mu_{2}-v_{2}\right)+\alpha+\beta} S_{j v_{1}+\beta, \xi\left(\mu_{1}-v_{1}\right)-\alpha-\beta} h_{1}(x),
\end{aligned}
$$

provided that $c_{22} c_{11}-c_{21} c_{12} \neq 0$, while $h_{2}(x)=-c_{11}^{-1} c_{12} h_{3}(x)$. The solution for $\phi_{1}$ and $\phi_{2}$ is then given by the representations (3.3) and (3.4).

4. Three pairs of dual equations. We shall now discuss the solution to the set of dual relations

$$
\begin{aligned}
S_{\ddagger \mu_{i}-\alpha, 2 \alpha}\left(\sum_{j=1}^{3} c_{i j} \phi_{j}(x)\right) & =p_{i}(x) & & \left(x \in I_{1}\right), \\
S_{\ddagger v_{t}-\beta, 2 \beta} \phi_{i}(x) & =0 & & \left(x \in I_{2}\right),
\end{aligned}
$$

where $i=1,2,3, I_{1}=(0,1)$ and $I_{2}=(1, \infty)$. 
Using the methods described in $\S 3$, we set

$\phi_{1}(x)=S_{\frac{1}{2} v_{1}+\beta, \frac{1}{2}\left(\mu_{1}-v_{1}\right)-\alpha-\beta} h_{1}(x)+S_{\frac{1}{2} v_{2}+\beta, \frac{1}{2}\left(\mu_{2}-v_{2}\right)-\alpha-\beta} h_{2}(x)+S_{\frac{1}{2} v_{3}+\beta, \frac{1}{2}\left(\mu_{3}-v_{3}\right)-\alpha-\beta} h_{3}(x)$,

$\phi_{2}(x)=S_{\frac{1}{2} v_{2}+\beta, \frac{1}{2}\left(\mu_{2}-v_{2}\right)-\alpha-\beta} h_{4}(x)$,

$\phi_{3}(x)=S_{\frac{1}{2} \nu_{3}+\beta, \frac{1}{2}\left(\mu_{3}-v_{3}\right)-\alpha-\beta} h_{5}(x)$,

where $h_{i}(x) \equiv 0, x \in I_{2}, i=1,2,3,4,5$.

It is a simple matter (by use of (2.13)) to verify that (4.3)-(4.5) satisfy the relations (4.2).

Substituting (4.3) into (4.1), for $i=1$, we obtain

$$
\begin{aligned}
I_{\frac{1}{2} v_{1}+\beta, \frac{1}{2}\left(\mu_{1}-v_{1}\right)+\alpha-\beta} & {\left[c_{11} h_{1}(x)\right]+S_{\frac{1}{2} \mu_{1}-\alpha, 2 \alpha} S_{\frac{1}{2} v_{2}+\beta, \frac{1}{2}\left(\mu_{2}-v_{2}\right)-\alpha-\beta}\left[c_{11} h_{2}(x)+c_{12} h_{4}(x)\right] } \\
& +S_{\frac{1}{2} \mu_{1}-\alpha, 2 \alpha} S_{\frac{1}{2} v_{3}+\beta, \frac{1}{2}\left(\mu_{3}-v_{3}\right)-\alpha-\beta}\left\{c_{11} h_{3}(x)+c_{13} h_{5}(x)\right\}=p_{2}(x) \quad\left(x \in I_{1}\right) .
\end{aligned}
$$

If we select $h_{2}(x), h_{3}(x), h_{4}(x), h_{5}(x)$ such that

we see that

$$
\left.\begin{array}{l}
c_{11} h_{2}(x)+c_{12} h_{4}(x)=0, \\
c_{11} h_{3}(x)+c_{13} h_{5}(x)=0,
\end{array}\right\}
$$

$$
h_{1}(x)=c_{11}^{-1} I_{\frac{1}{2} \mu_{1}+\alpha,-\frac{1}{2}\left(\mu_{1}-v_{1}\right)-\alpha+\beta} p_{1}(x) \quad\left(x \in I_{1}\right),
$$

provided that $c_{11} \neq 0$.

In the like manner, we find that substitution of (4.3)-(4.5) into (4.1), for $i=2$, yields, using the properties (2.6)-(2.12),

$$
\begin{aligned}
& c_{21} h_{2}(x)+c_{22} h_{4}(x) \\
& =F(x)-S_{\frac{1}{t} \mu_{2}-\alpha,-\frac{1}{2}\left(\mu_{2}-v_{2}\right)+\alpha+\beta} S_{\frac{1}{2} v_{3}+\beta, \frac{1}{2}\left(\mu_{3}-v_{3}\right)-\alpha-\beta}\left[c_{21} h_{3}(x)+c_{23} h_{5}(x)\right] \quad\left(x \in I_{1}\right),
\end{aligned}
$$

where

$$
F(x)=I_{\frac{1}{2} \mu_{2}+\alpha,-\frac{1}{2}\left(\mu_{2}-v_{2}\right)-\alpha+\beta} p_{2}(x)-c_{21} S_{\frac{1}{2} \mu_{2}-\alpha,-\frac{1}{2}\left(\mu_{2}-v_{2}\right)+\alpha+\beta} S_{\frac{1}{2} v_{1}+\beta, \frac{1}{2}\left(\mu_{1}+v_{1}\right)-\alpha-\beta} h_{1}(x),
$$

while substitution into (4.1), for $i=3$, gives us the relation

$$
\begin{aligned}
& c_{31} h_{3}(x)+c_{33} h_{5}(x) \\
& \quad=G(x)-S_{\frac{1}{2} \mu_{3}-\alpha,-\frac{1}{2}\left(\mu_{3}-v_{3}\right)+\alpha+\beta} S_{\frac{1}{2} v_{2}+\beta, \frac{1}{2}\left(\mu_{2}-v_{2}\right)-\alpha-\beta}\left[c_{31} h_{2}(x)+c_{32} h_{4}(x)\right] \quad\left(x \in I_{1}\right),
\end{aligned}
$$

with

$$
G(x)=I_{\frac{1}{2} \mu_{3}+\alpha,-\frac{1}{2}\left(\mu_{3}-v_{3}\right)-\alpha+\beta} p_{3}(x)-c_{31} S_{\frac{1}{2} \mu_{3}-\alpha,-\frac{1}{2}\left(\mu_{3}-v_{3}\right)+\alpha+\beta} S_{\frac{1}{2} v_{1}+\beta, \frac{1}{2}\left(\mu_{1}-v_{1}\right)-\alpha-\beta} h_{1}(x) .
$$

By making use of equations (4.6), we see that

$$
h_{5}(x)=\gamma_{1} G(x)-\gamma_{2} S_{\frac{1}{2} \mu_{3}-\alpha,-\frac{1}{2}\left(\mu_{3}-v_{3}\right)+\alpha+\beta} S_{\frac{1}{2} v_{2}+\beta, \frac{1}{2}\left(\mu_{2}-v_{2}\right)-\alpha-\beta} h_{4}(x),
$$

while

$$
h_{4}(x)=\gamma_{4} F(x)-\gamma_{5} S_{\frac{1}{2} \mu_{2}-\alpha,-\frac{1}{2}\left(\mu_{2}-v_{2}\right)+\alpha+\beta} S_{\frac{1}{2} v_{3}+\beta, \frac{1}{2}\left(\mu_{3}-v_{3}\right)-\alpha-\beta} h_{5}(x),
$$


where

$$
\begin{array}{ll}
\gamma_{1}=\frac{c_{11}}{c_{33} c_{11}-c_{13} c_{31}}, & \gamma_{2}=\frac{c_{32} c_{11}-c_{31} c_{12}}{c_{33} c_{11}-c_{13} c_{31}}, \\
\gamma_{4}=\frac{c_{11}}{c_{22} c_{11}-c_{12} c_{21}}, & \gamma_{5}=\frac{c_{23} c_{11}-c_{21} c_{13}}{c_{22} c_{11}-c_{21} c_{12}}
\end{array}
$$

We must, of course, make the assumption that

$$
c_{33} c_{11}-c_{13} c_{31} \neq 0 \text { and } \quad c_{22} c_{11}-c_{21} c_{12} \neq 0 \text {. }
$$

If we substitute (4.13) into (4.12) and make use of the fact that $I_{\eta, 0}$ is the identity operator, we find the following simple result for $h_{5}(x)$ :

$$
h_{5}(x)=\frac{\gamma_{1}}{1-\gamma_{2} \gamma_{5}} G(x)-\frac{\gamma_{2} \gamma_{4}}{1-\gamma_{\Sigma} \gamma_{5}} S_{\frac{1}{2} \mu_{3}-\alpha,-\frac{1}{2}\left(\mu_{3}-v_{3}\right)+\alpha+\beta} S_{\frac{1}{2} v_{2}+\beta, \frac{1}{2}\left(\mu_{2}-v_{2}\right)-\alpha-\beta} F(x) \quad\left(x \in I_{1}\right),
$$

provided that $\gamma_{2} \gamma_{5} \neq 1$.

Putting (4.14) into (4.13) we find that, for $x \in I_{1}, h_{4}(x)$ is given by

$$
\begin{aligned}
h_{4}(x)=\gamma_{4} F(x) & -\frac{\gamma_{1} \gamma_{5}}{1-\gamma_{2} \gamma_{5}} S_{\frac{1}{2} \mu_{2}-\alpha, \frac{1}{2}\left(\mu_{2}-v_{2}\right)+\alpha+\beta} S_{\frac{1}{2} v_{3}+\beta, \frac{1}{2}\left(\mu_{3}-v_{3}\right)-\alpha-\beta} G(x) \\
& \quad+\frac{\gamma_{2} \gamma_{4} \gamma_{5}}{1-\gamma_{2} \gamma_{5}} S_{\frac{1}{2} \mu_{2}-\alpha,-\frac{1}{2}\left(\mu_{2}-v_{2}\right)+\alpha+\beta}\left[S_{\frac{1}{2} \nu_{2}+\beta, \frac{1}{2}\left(\mu_{2}-\nu_{2}\right)-\alpha-\beta} F(x)\right] \\
= & \frac{\gamma_{4}}{1-\gamma_{2} \gamma_{5}} F(x)-\frac{\gamma_{1} \gamma_{5}}{1-\gamma_{2} \gamma_{5}} S_{\frac{1}{2} \mu_{2}-\alpha,-\frac{1}{2}\left(\mu_{2}-v_{2}\right)+\alpha+\beta} S_{\frac{1}{2} \nu_{3}+\beta, \frac{1}{2}\left(\mu_{3}-v_{3}\right)-\alpha-\beta} G(x),
\end{aligned}
$$

where $F(x)$ and $G(x)$ are given by (4.9) and (4.11) respectively, and we assume that $\gamma_{2} \gamma_{5} \neq 1$.

The results for $h_{2}(x)$ and $h_{3}(x)$ are given by (4.6) while the $\phi_{i}(i=1,2,3)$ are easily found from (4.3)-(4.5).

5. Examples. In this section we consider two examples of sets of simultaneous equations arising in the mathematical theory of elasticity. (See Sneddon and Lowengrub [8], Chapter 3, $\S 3.5$.) Both sets of equations appear in the solution to the problem of determining the stress field in the neighbourhood of a penny shaped crack in a solid under shear.

We first consider the set of equations

$$
\left.\begin{array}{rl}
\mathscr{H}_{0}\left[c_{11} A(\xi)+c_{12} B(\xi) ; \rho\right] & =1, \\
\mathscr{H}_{2}\left[c_{21} A(\xi)+c_{22} B(\xi) ; \rho\right] & =0,
\end{array}\right\} \quad(0<\rho<1),
$$


It is a simple matter to show that the above equations may be rewritten in the form

$$
\left.\left.\begin{array}{rl}
S_{0,0}\left[c_{11} A(\rho)+c_{12} B(\rho)\right] & =1, \\
S_{1,0}\left[c_{21} A(\rho)+c_{22} B(\rho)\right] & =0,
\end{array}\right\} \quad \begin{array}{rl}
\left(\rho \in I_{1}\right), \\
S_{-\frac{1}{2}, 1}[A(\rho)] & =0, \\
S_{\frac{1}{2}, 1}[B(\rho)] & =0,
\end{array}\right\} \quad\left(\rho \in I_{2}\right),
$$

where $I_{1}=(0,1)$ and $I_{2}=(1, \infty)$.

Following the methods indicated in $\S 3$, we see that

$$
\begin{aligned}
& A(\rho)=S_{\frac{1}{2}-\frac{1}{2}} h_{1}(\rho)+S_{\frac{1}{2},-\frac{1}{2}} h_{2}(\rho), \\
& B(\rho)=S_{\frac{1}{2},-\frac{1}{2}} h_{3}(\rho),
\end{aligned}
$$

where $h_{i}(\rho) \equiv 0, \rho \in I_{2}(i=1,2,3)$. Hence, from equation (3.6), we find that

and then

$$
h_{1}(\rho)=c_{11}^{-1} I_{0, \frac{1}{2}}[1]=c_{11}^{-1} \frac{2}{\sqrt{ } \pi} \quad\left(\rho \in I_{1}\right)
$$

$$
A(\rho)=c_{11}^{-1} \frac{2}{\sqrt{\pi}} \rho^{-\frac{1}{2}} J_{\frac{3}{2}}(\rho)
$$

while (3.7) yields

since

$$
h_{3}(\rho)=-\frac{c_{21}}{c_{22} c_{11}-c_{21} c_{12}} S_{1, \frac{1}{2}} S_{0,0}[1]=0
$$

$$
\int_{0}^{\infty} t^{\mu-v+1} J_{\mu}(a t) J_{v}(b t) d t=0 \quad \text { for } \quad 0<b<a
$$

and $\operatorname{Re} \nu>\operatorname{Re} \mu>-1$. (See Abramowitz [2], p. 487.)

Hence $h_{2}(\rho)=0$ and $B(\rho)=0$. We have assumed that $c_{11} \neq 0$.

For the second example, we consider the set of equations

$$
\begin{aligned}
& \mathscr{H}_{\mu_{i}}\left[\sum_{j=1}^{3} c_{i j} \phi_{j} ; \rho\right]=p_{i} \quad\left(\rho \in I_{1}, \quad i=1,2,3\right), \\
& \left.\begin{array}{rl}
\mathscr{H}_{0}\left[\xi^{-1} \phi_{1}(\xi) ; \rho\right] & =0, \\
\mathscr{H}_{2}\left[\xi^{-1} \phi_{2}(\xi) ; \rho\right] & =0, \\
\mathscr{H}_{1}\left[\xi^{-1} \phi_{3}(\xi) ; \rho\right] & =0,
\end{array}\right\} \quad\left(\rho \in I_{2}\right),
\end{aligned}
$$

where $p_{1}=1, p_{2}=p_{3} \equiv 0, I_{1}=(0,1)$ and $I_{2}=(1, \infty)$. 
Using the notation of $\S 2$, we may rewrite the above set as follows:

$$
\left.\begin{array}{rl}
S_{0,0}\left[\sum_{i=1}^{3} c_{1 i} \phi_{i}(\rho)\right] & =1, \\
S_{\frac{1}{2}, 0}\left[\sum_{i=1}^{3} c_{2 i} \phi_{i}(\rho)\right] & =1, \\
S_{1,0}\left[\sum_{i=1}^{3} c_{3 i} \phi_{i}(\rho)\right] & =0, \\
S_{-\frac{1}{2}, 1}\left[\phi_{1}(\rho)\right] & =0, \\
S_{\frac{1}{2}, 1}\left[\phi_{2}(\rho)\right] & =0, \\
S_{0,1}\left[\phi_{3}(\rho)\right] & =0,
\end{array}\right\} \quad\left(\rho \in I_{1}\right),
$$

Using the relations (4.3)-(4.5), we observe that

$$
\left.\begin{array}{l}
\phi_{1}(\rho)=S_{\frac{1}{2},-\frac{1}{2}} h_{1}(\rho)+S_{\frac{1}{3},-1} h_{2}(\rho)+S_{1,0} h_{3}(\rho), \\
\phi_{2}(\rho)=S_{\frac{5}{2},-1} h_{4}(\rho), \\
\phi_{3}(\rho)=S_{1,0} h_{5}(\rho),
\end{array}\right\}
$$

where on $I_{2}, h_{i}(\rho)=0(i=1,2,3)$, while on $I_{1}$ (see 4.14 and 4.15)

with

$$
\begin{aligned}
& h_{1}(\rho)=\frac{2 c_{11}^{-1}}{\sqrt{\pi}}, \\
& h_{5}(\rho)=\gamma_{1} G(\rho)-\gamma_{2} \gamma_{4} S_{1,0} S_{\frac{1}{2},-1} F(\rho), \\
& h_{4}(\rho)=\gamma_{4} F(\rho)-\gamma_{5} S_{\frac{1}{2}, 1} S_{1,0} h_{5}(\rho), \\
& h_{2}(\rho)=-\frac{c_{12}}{c_{11}} h_{4}(\rho), \quad h_{3}(\rho)=-\frac{c_{13}}{c_{11}} h_{5}(\rho),
\end{aligned}
$$

and

$$
F(\rho)=-c_{21} S_{\frac{1}{2}, 1} S_{\frac{1}{2},-\frac{1}{2}} h_{1}(\rho)=-\frac{c_{21}}{c_{11}} \frac{4}{\pi} \rho_{2} F_{1}\left(2, \frac{1}{2} ; 3 ; \rho^{2}\right) \quad(0<\rho<1)
$$

Hence

$$
G(\rho)=-c_{31} S_{1,0} S_{\frac{1}{2},-\frac{1}{3}} h_{1}(\rho)=0 \quad(0<\rho<1) .
$$

$$
\begin{array}{ll}
h_{5}(\rho)=-\frac{c_{21}}{c_{11}} \frac{2}{\sqrt{ }} S_{1,0} S_{\frac{1}{2},-\frac{1}{2}}[1]=0, \\
h_{3}(\rho)=0, \\
h_{4}(\rho)=-\gamma_{4} \frac{c_{21}}{c_{11}} \frac{4}{\pi} \rho_{2} F_{1}\left(2, \frac{1}{2} ; 3 ; \rho^{2}\right) \quad(0<\rho<1), \\
h_{2}(\rho)=-\gamma_{4} \frac{c_{12}}{c_{11}} \frac{4}{\pi} \rho_{2} F_{1}\left(2, \frac{1}{2} ; 3 ; \rho^{2}\right) \quad(0<\rho<1),
\end{array}
$$


while

and

$$
\begin{aligned}
& \phi_{2}(\rho)=-\gamma_{4} c_{21} S_{\frac{3}{2},-1} S_{\frac{1}{2}, 1}\left[2^{-\frac{1}{2}} \rho^{-\frac{1}{2}} J_{\frac{3}{2}}(\rho)\right]=-\frac{\gamma_{4} c_{21}}{\sqrt{2}} \rho^{-\frac{1}{2}} J_{\frac{2}{2}}(\rho), \\
& \phi_{3}(\rho)=0
\end{aligned}
$$

$$
\phi_{1}(\rho)=\sqrt{\frac{2}{\pi}} c_{11}^{-1}\left[1+\frac{c_{21}^{2}}{c_{22} c_{11}-c_{21} c_{12}}\right] \rho^{-\frac{1}{2}} J_{\frac{3}{2}}(\rho),
$$

where $\gamma_{4}=c_{11}\left(c_{22} c_{11}-c_{21} c_{12}\right)^{-1}$ and $\gamma_{1}, \gamma_{2}, \gamma_{3}$ and $\gamma_{5}$ are given in $\S 4$.

6. The general case. We shall discuss briefly the method of solving the set of $n$ equations given by (1.7) and (1.8). If we use the representation given for $S_{\eta, \alpha}$ in (2.5), we see that we wish to solve the set of dual equations given by

$$
\begin{aligned}
S_{\frac{1}{2} \mu_{i}-\alpha, 2 \alpha}\left[\sum_{j=1}^{n} c_{i j} \phi_{j}(x)\right] & =p_{i}(x) & \left(x \in I_{1}\right), \\
S_{\frac{1}{2} v_{i}-\beta, 2 \beta}\left[\phi_{i}(x)\right] & =0 & \left(x \in I_{2}\right),
\end{aligned}
$$

$(i=1,2, \ldots, n)$.

Suppose that we let

$$
\begin{aligned}
& \phi_{1}(x)=\sum_{i=1}^{n} S_{\frac{1}{2} v_{i}+\beta, \frac{1}{2}\left(\mu_{i}-v_{i}\right)-\alpha-\beta}\left[h_{i}(x)\right], \\
& \phi_{2}(x)=S_{\frac{1}{2} \nu_{2}+\beta, \frac{1}{2}\left(\mu_{2}-v_{2}\right)-\alpha-\beta}\left[h_{n+1}(x)\right] \text {, } \\
& \dot{\phi}_{i}(x)=S_{\frac{1}{2} v_{i}+\beta, \frac{1}{3}\left(\mu_{i}-v_{i}\right)-\alpha-\beta}\left[h_{i+n-1}(x)\right],
\end{aligned}
$$

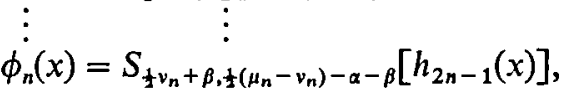

where $h_{i}(x)=0$ for $x \in I_{2}$. If we make use of the property (2.13), then we see that the representations (6.3) satisfy the equations given by (6.2).

Proceeding as in $\S \S 3$ and 4, we first substitute (6.3) into (6.1) for $i=1$. This yields

$$
\begin{aligned}
I_{\frac{1}{2} v_{1}+\beta, \frac{1}{2}\left(\mu_{1}-v_{1}\right)+\alpha-\beta} c_{11} h_{1}(x) & +S_{\frac{1}{2} \mu_{1}-\alpha, 2 \alpha} S_{\frac{1}{2} v_{2}+\beta, \frac{1}{1}\left(\mu_{2}-v_{2}\right)-\alpha-\beta}\left[c_{11} h_{2}(x)+c_{12} h_{n+1}(x)\right] \\
& +\ldots+S_{\frac{1}{2} \mu_{1}-\alpha, 2 \alpha} S_{\frac{1}{2} v_{t}+\beta, \frac{1}{2}\left(\mu_{i}-v_{i}\right)-\alpha-\beta}\left[c_{11} h_{i}(x)+c_{1 i} h_{n+(i-1)}(x)\right] \\
& +\ldots+S_{\frac{1}{1} \mu_{1}-\alpha, 2 \alpha} S_{\frac{1}{2} v_{n}+\beta, \frac{1}{2}\left(\mu_{n}-v_{n}\right)-\alpha-\beta}\left[c_{11} h_{n}(x)+c_{1 n} h_{2 n-1}(x)\right] \\
& =p_{1}(x) \quad\left(x \in I_{1}\right) .
\end{aligned}
$$


If we select the $h_{i}(x), x \in I_{1}$ such that

$$
\left.\begin{array}{c}
c_{11} h_{2}(x)+c_{12} h_{n+1}(x)=0 \\
c_{11} h_{3}(x)+c_{13} h_{n+2}(x)=0 \\
\vdots \\
\vdots \\
c_{11} h_{i}(x)+c_{1 i} h_{n+(i-1)}(x)=0 \\
\vdots \\
c_{11} h_{n}(x)+c_{1 n} h_{2 n-1}(x)=0,
\end{array}\right\}
$$

then we find that $h_{1}(x)$ for $x \in I_{1}$ is given by

$$
h_{1}(x)=c_{11}^{-1} I_{\frac{1}{2} \mu_{1}+\alpha,-\frac{1}{2}\left(\mu_{1}-v_{1}\right)-\alpha+\beta} p_{1}(x),
$$

provided that $c_{11} \neq 0$.

The remainder of the analysis proceeds in precisely the same way as in the case $n=3$. The relations (6.3) are first systematically substituted into the remaining $n-1$ relations in (6.1) and then, using (6.5) and the various properties of the $I$ operator, we obtain representations for linear combinations of $h_{i}(x)$ and $h_{n+(i-1)}(x)$. By use of (6.4) and these representations we can obtain the $h_{i}(x)$, for $x \in I_{1}$, after noting the same cancellation of operators that occurs in $\S 4$. These results are extremely cumbersome and will not be reproduced here. For most mixed boundary value problems, the results for $n=2$ and $n=3$ will suffice. It should be pointed out that careful use of the $S$ operator is necessary, for, since we do not know the component of $p_{i}(x)$ for $x \in I_{2}$, we cannot evaluate $S_{\eta, \alpha} p_{i}(x)$.

By making similar representations as in (6.3), the problem of finding solutions to the set of equations (1.3) and (1.4) with any continuous functions $w_{i j}(t), v_{i j}(t)$ is reduced to that of solving a simultaneous system of Fredholm equations with a singular kernel. This is briefly discussed in $\S 4$ of the Erdogan and Bahar [4] paper. We might just add that for these problems it is best to let the $\phi_{i}$ 's be represented as follows:

$$
\phi_{i}(x)=S_{\frac{1}{2} v_{i}+\beta, \frac{1}{2}\left(\mu_{i}-v_{i}\right)-\alpha-\beta} h_{i}(x) \quad(i=1,2, \ldots, n),
$$

where $h_{i}(x) \equiv 0$ for $x \in I_{2}$.

\section{REFERENCES}

1. M. Abramowitz and I. A. Stetgun, Handbook of mathematical functions (Nat. Bureau of Standards, App. Math. Series 55, 1964). 21-24.

2. E. T. Copson, On certain dual integral equations, Proc. Glasgow Math. Assoc. 5 (1961),

3. A. Erdelyi and I. N. Sneddon, Fractional integration and dual integral equations, Canad.J. Math. 14 (1962), 685-693.

4. F. Erdogan and L. Y. Bahar, On the solution of simultaneous dual integral equations, S.I.A.M. Journal 12 (1964), 666-675.

5. M. Lowengrub and I. N. Sneddon, The solution of a pair of dual integral equations, Proc. Glasgow Math. Assoc. 6 (1963), 14-18.

6. A. S. Peters, Certain dual integral equations and Sonine's integral (Technical report Imm-NYU 285, Inst. Math. Sci. NYU August, 1961). 
7. I. N. Sneddon, Mixed boundary value problems of potential theory (North Holland Publishing Co. Amsterdam, 1966).

8. I. N. Sneddon and M. Lowengrub, Crack problems in the classical theory of elasticity (New York, 1968).

9. C. J. Tranter, Integral Transforms in Mathematical Physics (New York, 1956).

10. R. A. Westmann, Simultaneous pairs of dual integral equations, SIAM Rev. 7 (1965), 341-348.

DUKE UNIVERSITY

UNIVERSITY OF GLASGOW

Permanent Address: Department of Mathematics, Indiana University, Bloomington, Indiana 